\title{
Labyrinthe
}

$35 \mid 2010$ (2)

Empire Reader

\section{Entretien avec Ivan Segré}

La philosophie française et les « Juifs »

\section{Basile Dewez et Ivan Segré}

\section{(2) OpenEdition}

Journals

Édition électronique

URL : http://journals.openedition.org/labyrinthe/4092

DOI : $10.4000 /$ labyrinthe.4092

ISSN : 1950-6031

Éditeur

Hermann

\section{Édition imprimée}

Date de publication : 28 août 2010

Pagination : 117-132

ISBN : 9782705669973

Référence électronique

Basile Dewez et Ivan Segré, «Entretien avec Ivan Segré », Labyrinthe [En ligne], 35 | 2010 (2), mis en ligne le 27 juillet 2012, consulté le 21 avril 2019. URL : http://journals.openedition.org/labyrinthe/4092 ; DOI : 10.4000/labyrinthe.4092 


\title{
Entretien avec Ivan Segré
}

\section{La philosophie française et « les Juifs »}

\author{
Propos recueillis par Basile Dewez
}

Ivan Segré est docteur en philosophie. Il vit aujourd'hui en Israël, où il mène en parallèle études philosophiques et talmudiques. En 2009, il a publié conjointement aux éditions Lignes La réaction philosémite ou La trahison des clercs et Qu'appelle-t-on penser Auschwitz (ce dernier avec une préface d'Alain Badiou).

Basile Dewez est doctorant au Centre Raymond-Aron de recherches politiques de l'École des hautes études en sciences sociales. Sa recherche porte sur la société israélienne et le système politique israélien, et en particulier la question de ce que désigne la définition d'Israël comme État juif et démocratique. Il a été chercheur invité à l'Université hébraïque de Jérusalem.

\section{Basile Dewez : Quelles sont les circonstances qui ont présidé à la rédaction de vos deux ouvrages?}

Ivan Segré : Mon travail de recherche en philosophie a porté principalement sur la « question juive », plus précisément sur ses reformulations contemporaines. Je me suis proposé d'éclaircir, en écrivant ces deux ouvrages, la manière dont la « question » se pose aujourd' hui dans la philosophie contemporaine française. Avec Qu'appelle-t-on penser Auschwitz, le premier volet du diptyque paru aux éditions Lignes à l'initiative de l'éditeur Michel Surya, j'aborde la question du silence de Heidegger sur l'extermination des Juifs, les analyses d'Arendt sur le procès d'Eichmann à Jérusalem, enfin la manière dont Philippe Lacoue-Labarthe s'est attaché à penser ce qu'il appelle « la signification métaphysique » de l'extermination des Juifs dans les chambres à gaz nazies. Mais j'aborde également les positions d'Alain Badiou, de Jean-Claude Milner, de Benny Lévy, d'Alain Finkielkraut ou d'Éric Marty au sujet d'Auschwitz, des Juifs, de 
l'antisémitisme et du négationnisme. Avec La réaction philosémite, le second volet, je m'intéresse à l'aspect plus immédiatement idéologique de la question. L'apparition d'un courant intellectuel français qui, au nom de la « défense d'Israël » et de la « lutte contre l'antisémitisme », a développé un argumentaire réactionnaire, contre les Maghrébins ou les Noirs d'identité musulmane, plus largement contre les jeunes des quartiers populaires, et contre les progressistes, est un phénomène notoire. L'originalité de mon analyse, c'est de montrer que, à y bien regarder, ce courant intellectuel français n'est absolument pas le symptôme d'un « repli communautaire juif », comme on a pu le penser, mais plutôt l'avant-garde d'une réaction idéologique dont le mot d'ordre véritable est la défense de l'Occident, et non la défense des Juifs ou d'Israël.

\section{Basile Dewez : En quel sens dites-vous que la «question juive » s'est posée à la philosophie contemporaine ?}

Ivan Segré : Avant d'en arriver au contemporain, il est nécessaire de faire un peu d'histoire de la philosophie. À la fin du XVIII ${ }^{e}$ siècle et au début du XIX ${ }^{\mathrm{e}}$, avec les Lumières et les transformations politiques qui leur sont corrélatives, la question de l'émancipation des Juifs s'est posée en Europe, et principalement en Allemagne, où la « question juive », résolue d'emblée en France du fait de l'axiome égalitaire des révolutionnaires, est demeurée problématique, discutée jusque tard dans le $\mathrm{XIX}^{\mathrm{e}}$ siècle - d'où le texte de Marx, qui date de $1843^{1}$. Mais notons bien que Die Judenfrage, La question juive, contrairement à ce que l'on dit souvent, n'est pas un texte de Marx. C'est un texte que publie cette même année 1843 le philosophe allemand Bruno Bauer, hégélien, protestant, exégète de la Bible et des Évangiles. Marx a écrit, lui, une critique des textes de Bauer, qu'il a intitulée : Zur Judenfrage, qu'on pourrait traduire : À propos de «la question juive » telle que la pose Bruno Bauer, car « la question juive », dans le texte de Marx, est à mettre entre guillemets. Le propos de Marx est en effet de soutenir, contre Bauer, qu'il n'y a précisément pas de « question juive », mais une autre question, absolument distincte, qui est la question de l'émancipation politique et sociale. Donc poser

1. Voir le numéro de Labyrinthe consacré aux attitudes juives critiques face à l'émancipation : «Des Juifs contre l'émancipation. De Babylone à Benny Lévy », dir. Pierre Savy et David Schreiber, Labyrinthe. Atelier interdisciplinaire, 28, 2007. [NDLR] 
une « question juive », selon Marx, c'est une manière d'occulter la vraie question. Que dit Bauer, en substance ? Il dit que les revendications des Juifs visant à obtenir des droits civiques en Allemagne sont infondées, et le resteront tant que les Juifs n'auront pas aboli tout rapport subjectif contraignant à la religion juive, car on ne peut être le sujet de deux lois non seulement distinctes, mais contradictoires, l'une juive, archaïque et particulariste, l'autre politique, moderne et universaliste - ou chrétienne, c'est égal selon Bauer. Marx lui répond que la distinction entre la sphère publique et la sphère privée est un acquis du libéralisme politique, et que par conséquent les Juifs témoignent d'une conscience politique moderne en revendiquant des droits civiques, tandis que l'État prussien témoigne d'une conscience politique archä̈que en les lui refusant. Citons Marx : « Si l'État évangélise lorsque, bien qu'État, il agit chrétiennement à l'égard des Juifs, le Juif fait de la politique lorsque, bien que Juif, il réclame des droits civiques ». Marx prend ainsi position, contre Bauer, pour les droits civiques des Juifs en tant que Juifs, et ce au nom de la modernité politique, en quelque sorte. Mais d'un autre côté, Marx considère que le libéralisme politique n'est qu'une étape, nécessaire mais pas suffisante, et qu'avec l'avènement du communisme l'homme sera libéré de toute forme de sujétion, qu'elle soit religieuse, politique, économique ou sociale, ce par quoi il rejoint Bauer, du moins en ce qu'il juge, lui aussi, que la religion juive est un archaïsme. Mais, ajoute Marx, la religion chrétienne autant que la juive. La lecture qui consiste à présenter Marx comme un penseur antijuif, voire antisémite avant l'heure, repose donc sur une occultation du contenu patent de ses écrits, pour n'en retenir que le contenu latent, lequel, c'est vrai, n'est pas sans reproduire quelques uns des préjugés antijuifs de Bauer. Mais n'oublions cependant pas que ces préjugés étaient à l'époque la chose du monde la mieux partagée en Allemagne et ailleurs.

Faisons maintenant un saut d'un siècle. Au sortir de la Seconde Guerre mondiale, Jean-Paul Sartre écrit ses Réflexions sur la question juive. Ce faisant, Sartre s'inscrit très distinctement dans la filiation philosophique de Marx, puisqu'il s'agit une nouvelle fois de faire la critique du préjugé selon lequel il y aurait une «question juive », et d'opposer au préjugé un axiome égalitaire ( Pas un Français ne sera libre tant que les Juifs ne jouiront pas de la plénitude de leurs droits » conclut Sartre). Mais contrairement à Marx, qui s'attache à montrer comment la critique - par ailleurs légitime selon lui - du judaïsme religieux détourne la pensée révolutionnaire de son objet, qui est la critique de la société bourgeoise, 
Sartre ne s'en tient pas là, et propose en outre une critique du préjugé antijuif en tant que tel, voire une sorte de phénoménologie de ce préjugé. On connaît la thèse de Sartre : le « Juif » est une invention de l'antisémite. Cette thèse aujourd'hui nous étonne : comment peut-on soutenir que le « Juif » est une invention de l'antisémite, autrement dit que le mot « juif » n'a d'autre acception signifiante que celle d'un individu quelconque auquel on prête des caractéristiques péjoratives, des singularités repoussantes? Sartre ne connaissait rien du judaïsme ou des Juifs ; en un sens, son objet n'était pas cela, mais précisément le préjugé antisémite. Son propos était de montrer que le préjugé antisémite est une invention qui ne nous dit rien du Juif, mais en revanche nous renseigne sur l'antisémite qui produit un tel imaginaire. L'insuffisance du texte de Sartre à nos yeux, c'est-à-dire après plus d'un demi-siècle de recul, d'analyse et de réflexion sur les événements de la Seconde Guerre mondiale, tient pourtant en deux points : 1) Sartre ne mentionne aucunement l'extermination des Juifs par les nazis, ni le génocide, ni les camps, ni les chambres à gaz ; 2) il n'établit aucun rapport entre l'animosité contre les Juifs et l'existence propre, autonome des Juifs. Je m'explique sur ce second point : si l'antisémite produit un «Juif » imaginaire, caricatural, abject, reste que les Juifs existent indépendamment de l'antisémite. Or si l'on prend acte de ceci que, avec le nazisme, le préjugé antisémite s'est donné une consistance politique inouïe en exterminant physiquement les Juifs, on ne peut plus s'en tenir aux analyses de Sartre écrites dans l'immédiat après-guerre. Pourquoi ? Parce que si l'on s'en tient là, on risque de reproduire en idée la politique effective de destruction des Juifs, dès lors que réduire le Juif à l'imaginaire de l'antisémite, c'est une manière de nier l'existence autonome, signifiante par soi, des Juifs. Autrement dit cette existence autonome, signifiante par soi, doit être reconnue comme telle, pour que le «Juif » ne soit pas en quelque sorte condamné à disparaître avec l'imaginaire antisémite qui le produit. Mais on se retrouve alors dans une situation délicate, car jusque-là, celui qui attribuait aux Juifs des caractéristiques signifiantes, singulières, c'était précisément l'antisémite! La question posée à quiconque s'emploie à penser Auschwitz, ou « après Auschwitz », est ainsi devenue : comment reconnaître une signification au fait juif qui puisse nous éclairer sur la pulsion de mort dont les Juifs ont été l'objet, la cible, sans tomber dans un double écueil, qui serait ou bien d'entrer dans la fable antisémite, laquelle donne aux Juifs une consistance négative, ou bien d'entrer dans « la fable 
religieuse » - comme l'écrit Badiou au sujet de ceux qu'il appelle les « sacralisateurs du nom juif »-, laquelle donnerait bien une consistance positive aux Juifs, mais, d'un point de vue philosophique, au prix fort, si je puis dire, puisque cela reviendrait à s'abandonner à cette illusion religieuse dont Freud, après Marx, avait prédit l'avenir...

Vous voyez que ce petit préambule historique est éclairant, puisqu'on comprend aussitôt que le sionisme étatique est précisément apparu comme la réponse à cette question, en affirmant : l'existence signifiante par soi des Juifs est leur souveraineté étatique en Palestine. Et de là à conclure que l'antisionisme est la forme nouvelle de l'antisémitisme, il n'y a évidemment qu'un pas, du moins si l'on concède qu'il n'est nulle autre réponse à cette question que l'État d'Israël. En effet, selon ce point de vue, quiconque met en cause l'existence de l'État d'Israël met par là même en cause l'existence signifiante par soi des Juifs. Autrement dit, l'intellectuel antisioniste se proposerait au fond, et ce dans le meilleur des cas, de réduire le « Juif » à une construction imaginaire issue du préjugé antisémite, ce pourquoi, sous le couvert d'une dénonciation de principe de l'antisémitisme, l'antisioniste reproduirait en idée la négation effective des Juifs par les nazis. Et vous avez là résumé en quelques mots l'essentiel des critiques qui ont été faites au livre de Badiou Circonstances, 3. Portées du mot «juif». Mes deux ouvrages consistent notamment à proposer un autre regard sur la question.

Basile Dewez : Dans Qu'appelle-t-on penser Auschwitz, vous convoquez en effet, outre Heidegger et Arendt, les philosophes contemporains Lacoue-Labarthe et Badiou, et brossez en creux le portrait d' « antiphilosophes » contemporains comme Marty, Finkielkraut, Milner ou Benny Lévy. Quel est l'enjeu du différend entre philosophes et « antiphilosophes", et pourquoi ce différend se concentre-t-il aujourd'hui sur le nom « juif »?

Ivan Segré : Il faut ici distinguer trois termes : philosophie, antiphilosophie et rhétorique (ou sophistique). Depuis Platon, le philosophe bataille avec son autre mimétique, imaginaire ou faussaire qu'est le rhéteur. Pour résumer les choses d'un mot, disons que le philosophe s'emploie à penser rationnellement l'Idée de la justice, tandis que le rhéteur s'emploie à démontrer tout aussi rationnellement qu'il n'y a pas d'Idée de la justice, et que, en guise de justice, il convient de se soumettre à ce que les puissants 
du jour auront décrété juste. L'antiphilosophe s'oppose de manière tout aussi radicale au philosophe, mais avec cette différence de poids qu'il est, lui, pour reprendre un mot de Sartre, « irrécupérable » par les puissants du jour. Ainsi Kierkegaard, ainsi Nietzsche, ainsi Wittgenstein, ainsi Lacan, pour citer les grands noms de ce que l'on conviendra d'appeler, après Lacan, l'antiphilosophie. Mais également Heidegger, dont la pensée de la « fin de la métaphysique » est une forme d'antiphilosophie, en ce qu'il s'agit de faire advenir une autre pensée que celle de la rationalité philosophique telle qu'elle s'est fondée avec Platon. Lacoue-Labarthe n'est donc précisément pas philosophe, puisqu'il s'inscrit dans la pensée d'une fin de la philosophie, tandis que Badiou, lui, est philosophe, réfutant qu'il y ait une fin de la philosophie, et le réfutant de la manière la plus claire et distincte qui soit en systématisant une philosophie, précisément. J'ai, dans mes ouvrages, montré en quoi Benny Lévy, Milner ou Marty, avaient quitté l'antiphilosophie pour la rhétorique dans leur polémique avec le philosophe Badiou. Quant à Finkielkraut, ou Bernard-Henri Lévy, je ne vois pas qu'on puisse leur conférer le titre de philosophe, ou d'antiphilosophe, sans perdre aussitôt toute crédibilité. Une fois les personnages introduits, venons-en à l'intrigue proprement dite. Mais auparavant, précisons encore un point d'histoire, j'entends un point d'histoire de la philosophie française d'après-guerre.

La représentation de l'après-guerre comme un « après Auschwitz » n'a pas été immédiate, loin de là. C'est pourquoi on ne peut du reste reprocher à Sartre ses omissions sans faire preuve d'un certain anachronisme. Rappelons-nous que Jules Isaac et Léon Poliakov, les initiateurs de l'historiographie d'un antisémitisme occidental ayant abouti au génocide nazi, se sont d'abord heurtés à l'incompréhension, voire à la franche hostilité des institutions universitaires et plus largement intellectuelles françaises. L'antisémitisme, comme les chambres à gaz, étaient alors un « détail » de l'Histoire pour à peu près tout le monde. C'est à partir de la fin des années soixante-dix et surtout dans le courant des années quatre-vingts que l'on voit apparaître une véritable réflexion sur ce qu'a été l'antisémitisme nazi et, partant de là, sur ce qu'il importe de penser quant au fait de l'existence des Juifs, quant à ce que j'ai appelé la signifiance de ce fait. Dans le domaine littéraire, on voit apparaître le motif positif du «Juif » dans l'œuvre de Blanchot, ainsi que dans celle de Duras. Yann Andrea Steiner de Marguerite Duras, publié en 1982, est peut-être l'entreprise poétique la plus radicale quant à la pensée de l'existence signifiante des Juifs, et 
quant à la pensée du crime nazi. Citons la conclusion : «C'était peut-être quelque chose d'encore inconnu, Théodora Kats, un nouveau silence de l'écriture, celui des femmes et des Juifs ». L'œuvre de Paul Celan, qui s'est donné la mort en 1970, commence également à apparaître pour ce qu'elle est, une révélation poétique, non sans rapport, évidemment, avec Auschwitz, et avec la pensée de la signifiance du mot « juif ». La rencontre entre Paul Celan et Martin Heidegger devient alors un motif saisissant de la question posée à la pensée philosophique par le mot « juif ». En 1987, Lacoue-Labarthe publie La fiction du politique, et Lyotard Heidegger et "les Juifs », deux ouvrages qui abordent la question de l' " intolérable silence » de Heidegger quant à l'extermination des Juifs, et exposent ce en quoi il importe, pour la philosophie, de penser la singularité du crime nazi. Par ailleurs, à cette même époque, la pensée de Lévinas commence à acquérir la renommée qu'on lui connaît aujourd'hui. Il est notamment invité à la Sorbonne pour donner une série de conférences. Il faut mesurer à quel point la présence de Lévinas à la Sorbonne est en soi une révolution des esprits, puisque l'œuvre de Lévinas est une réponse non philosophique aux questions de la philosophie, ou de l'ontologie, ce qu'il appelle une éthique, puisant dans les textes traditionnels juifs l'inspiration d'une pensée affirmant la primauté de l'éthique sur l'ontologique. C'est aussi l'époque où les psychanalystes commencent à soupçonner qu'ils mettent au jour d'antiques vérités, notamment talmudiques. Il y a enfin, au crépuscule de la vie de Sartre, en 1980, son dialogue avec Benny Lévy, au cours duquel il revient sur ses Réflexions sur la question juive et affirme leur insuffisance, car les Juifs existent bien indépendamment du fantasme antisémite. Il y a une « histoire juive » dit Sartre, qui est à penser en tant que telle. À ce propos, il importe de souligner un point : dans son dialogue avec Benny Lévy, la reconnaissance par Sartre d'une histoire juive, signifiante par soi, a précisément pour enjeu de sortir de la philosophie hégélienne de l'histoire, c'est-à-dire de penser un sens de l'existence collective qui ne soit pas homogène à la philosophie de l'État. Il apparaît donc clairement que le trait caractéristique de ces diverses pensées de la consistance positive, signifiante par soi des Juifs, de Sartre à Duras en passant par Lacoue-Labarthe ou Lyotard, n'est nullement homogène au sionisme étatique. Autrement dit, pour tous ces penseurs, la réponse à la question posée après Auschwitz n'est pas l'État d'Israël, ce qui ne signifie pas qu'ils soient antisionistes - Lévinas et Celan, par exemple, étaient attachés à l'existence de l'État d'Israël -, mais que cette 
question est annexe eu égard à leur préoccupation essentielle, qui est de penser l'existence signifiante des Juifs indépendamment de la question d'un État « juif », indépendamment de la philosophie de l'histoire telle que la concevait Hegel.

La position de Badiou, à cet égard, est très intéressante et singulière, parce que, d'une part, il s'inscrit dans la continuité de ce mouvement de pensée, qui vise à conférer aux Juifs une positivité en tant que telle ; et que, d'autre part, il rompt avec lui, puisque Célan, Blanchot, Duras, Lévinas, Lyotard, Lacoue-Labarthe, Benny Lévy ou encore Milner développent une pensée hors philosophie. Badiou, lui, se propose de penser les «portées du mot "juif" » en philosophe rationaliste, platonicien. Et c'est là son incontestable singularité. Que dit Badiou des « portées du mot "juif" », pour reprendre le titre de son Circonstances, 3, recueil de textes écrits entre 1982 et nos jours? Sa thèse est que la figure signifiante du Juif, aux yeux du philosophe qu'il est, est celle du « Juif errant », parce que l'errance est ce qui délivre l'individu des déterminations bornées, identitaires, communautaires ou nationales, et le rend disponible, prêt à répondre à la vocation du sujet humain, qui est de n'avoir d'autre loi que celle des vérités, singulières, universelles, éternelles. Et c'est là ce qui rendrait raison du fait historique de la surreprésentation des Juifs dans l'aventure des procédures de vérités : la psychanalyse de Freud, la musique atonale de Schoenberg, le montage cinématographique d'Eisenstein, les mouvements d'émancipation politique, ou encore les avancées scientifiques décisives de la modernité, etc. Enfin c'est là ce qui expliquerait la politique nazie d'extermination des Juifs, car au travers des Juifs, ce qu'auraient visés les nazis, c'est le sujet des vérités, artistiques, politiques, scientifiques. L'existence du Juif étant signifiante, essentielle aux yeux du philosophe platonicien, en tant que, « errant », il est comme voué aux vérités universelles, Paul de Tarse apparait aux yeux de Badiou comme la figure du Juif par excellence, puisque saint Paul déclare, contre les conservatismes religieux, qu'une vérité n'est telle qu'à la condition d'être égale pour tous. Autre paradoxe, qui nous reconduit cette fois dans le contemporain, et qui est la conséquence logique du premier : il faudrait, en tant que Juif, prononcer l'abolition de l'État d'Israël comme État « juif » et déclarer qu'il n'est de politique qu'égale pour tous, sans distinction identitaire d'aucune sorte. D' où la conclusion qu' on a tirée que Badiou est un philosophe « antisémite », puisqu'il récuse tant la forme religieuse juive que sa forme politique nationale, à savoir l'État d'Israël. Le malentendu 
était sans doute inévitable. Et pourtant, non seulement Badiou n'est pas « antisémite », mais il est le seul philosophe, à ma connaissance, à avoir affirmé et pensé une articulation essentielle entre le nom «juif» et la vraie philosophie, disons l'Idée platonicienne. Or ce point me parait être beaucoup plus décisif, signifiant, que ne le sont ses positions à l'égard de l'État d'Israël. La thèse de Badiou sur le « Juif errant » ouvre en effet une brèche dans l'espace de la philosophie occidentale, en ce sens que par sa thèse sur les portées du mot «juif », Badiou nous rappelle, consciemment ou inconsciemment, que le personnage qui incarne le commencement inaugural de la philosophie, Socrate, mis en scène par Platon, n'est peut-être pas aussi grec que cela, précisément... Nietzsche tient du reste que Platon s'est instruit auprès des sources juives lors de son voyage en Égypte; et cette thèse est également soutenue dans des textes traditionnels juifs du Moyen Âge, qu'il s'agisse de Platon ou de Pythagore. Mais surtout, le dernier texte de Platon, Le Sophiste, met en scène un autre personnage en lieu et place de Socrate, ultime personnage que Platon nomme l'Étranger, autrement dit l'autre de la cité grecque. Pour qui sait lire, Badiou soutient la thèse que l'Étranger introduit par Platon dans cet ultime dialogue, l'autre de la cité grecque, c'est le Juif errant. Badiou écrit dans Logiques des mondes, au sujet d'un texte de Malraux sur l'Idée de la peinture telle qu' on peut en suivre la trace depuis les grottes de Lascaux jusqu'à Picasso : «J'aime cette comparaison des vérités éternelles, tel qu'elles procèdent et renaissent dans le devenir, avec le Juif errant. » Les contradicteurs du philosophe, comme Marty, Milner ou Benny Lévy, ont voulu dresser de lui le portrait d'un philosophe antijuif, ou d'un négateur du fait juif, parce qu'il critique l'idée d'un État « juif » en se revendiquant d'un axiome politique égalitaire, ou bien parce qu'il voit dans le « Juif Paul », comme Badiou l'appelle, un fondateur de l'universalisme, ou encore parce qu' au nom du rationalisme, il disqualifie tout recours à une transcendance religieuse. Mais ce faisant, ils ont radicalement occulté l'apport de Badiou à notre « question juive », et aussi ses précieux éclairages sur la question « Heidegger».

\section{Basile Dewez : Justement, comment expliquer le silence de Heidegger sur l'extermination des Juifs ?}

Ivan Segré : Cette question, qui est au centre de mon ouvrage, est presque un point de fixation dans la philosophie française d'après- 
guerre. Heidegger appelle à revenir au fondement de ce qui détermine en vérité la pensée occidentale, ce qui suppose selon lui de prononcer la fin de la métaphysique, autrement dit du platonisme, et de ressaisir le sens inaugural de l'antique parole grecque, pré-socratique, où notre destin, c'est-à-dire le destin de l'Occident, serait comme en attente. Le Juif, dans cette affaire, est donc absolument insignifiant. Pire, il n'est pas sans participer de ce qui oblitère le retour à la parole inaugurale grecque, laquelle serait, en quelque sorte, indemne du monothéisme juif ; d'où ceci qu'au regard de l'essentiel, on peut bien fermer les yeux sur les vulgarités antisémites des nazis, puis après la guerre, et jusqu'à sa mort, considérer que de l'extermination des Juifs, il n'y a précisément rien à dire qui concerne l'essentiel. Ce à quoi Lacoue-Labarthe répond, ou objecte à Heidegger que sa faute est justement de n'avoir pas su, ou voulu voir que l'extermination des Juifs concerne l'essentiel. Pourquoi ? Parce que c'est au travers de l'extermination par chambres à gaz que la métaphysique occidentale, ou l'onto-théologie occidentale, se révèle être ce que Heidegger enseigne qu'elle est, à savoir un nihilisme essentiel, une oblitération de la question du sens de l'être. Et si Heidegger n'a pas voulu voir qu'il n'était nullement indifférent que les chambres à gaz servent précisément à l'extermination des Juifs, c'est parce que du même coup, nous dit Lacoue-Labarthe, le philosophe allemand, occidental, aurait été sommé de penser le rapport entre un nihilisme essentiel et le meurtre des Juifs. Dans sa Fiction du politique, Lacoue-Labarthe conclut : "Dieu est effectivement mort à Auschwitz, en tout cas le Dieu de l'Occident gréco-chrétien, et ce n'est par aucune sorte de hasard que ceux que l'on voulait anéantir étaient les témoins, dans cet Occident-là, d'une autre origine du Dieu qui y avait été vénéré et pensé - si ce n'est même, peutêtre, d'un autre Dieu, resté libre de sa captation hellénistique et romaine et entravant par là même le programme de l'accomplissement. C'est pourquoi cet événement, l'Extermination, est à l'égard de l'Occident la terrible révélation de son essence ».

En regard de ce que j'ai appelé la «thèse » de Lacoue-Labarthe, la position de Badiou est en apparence rigoureusement adverse, puisqu'il récuse toute fin de la métaphysique, de même qu'il récuse que la métaphysique soit occidentale, et de même enfin qu'il récuse que la métaphysique ait quelque rapport que ce soit avec l'extermination des Juifs par les nazis. C'est du reste en raison de cette triple récusation que Marc Goldschmidt, dans un article paru dans Les Temps modernes en 
2009, s'en prend à Badiou, qu'il présente comme un penseur de « la fin de l'exception juive », autrement dit un penseur qui s'évertuerait à faire comme si de rien n'était, comme si rien n'avait eu lieu, comme si l'on pouvait continuer à philosopher comme avant, après le nazisme, après Auschwitz, après Derrida, Lyotard ou Lacoue-Labarthe. Marc Goldschmidt ne se rend pas compte, me semble-t-il, qu'il pose en fait la question suivante : comment peut-on être platonicien après Auschwitz ? Eh bien tâchons de l'éclairer sur ce point : si Heidegger en appelle à revenir, pardelà Platon, à la parole inaugurale grecque, c'est précisément parce que l'Idée de Platon n'est pas une telle parole inaugurale grecque, qu'elle est autre que le commencement grec, qu'elle est autre que la révélation grecque, autre que le dieu grec, au sens où Heidegger a conclu, au terme de sa méditation : "seul un dieu peut nous sauver». Nietzsche, dans son acharnement anti-platonicien, situe l'origine autre que grecque de l'Idée dans le Temple de Jérusalem, autrement dit dans la prêtrise juive, ce qui n'est pas exactement un compliment sous sa plume. Badiou la situe dans le geste singulier, éternel, qui consiste à penser, à se tourner vers l'Idée, à convertir son regard, lit-on dans certaines traductions du texte platonicien, ou encore, traduit pour sa part Badiou, à se tourner « vers ce qui de l'étant est sa face exposée à l'Idée ». C'est ainsi qu'on devient l'Étranger, l'autre du sophiste, l'autre de la cité grecque, l'autre de l'animal humain, social, assujetti au règne des opinions, et qui est allé, comme on sait, en bon démocrate, voter la condamnation à mort de Socrate, comme d'autres votèrent pour le Parti national-socialiste. Or cette altérité, qui est celle de tout sujet humain vivant sous la contrainte émancipatrice de l'Idée, Badiou nous dit que c'est précisément cela, l'existence signifiante par soi des Juifs, la véritable portée, à la fois singulière et universelle, du mot « juif ». Et il a absolument raison ; sauf que ce n'est pas le «Juif Paul » qui en est l'incarnation, ou le modèle fondateur, ni même Socrate, mais le maître du Talmud, exemplairement Rabbi Shimon bar Yohaï qui, à l'inverse de Paul finissant ses jours dans les coulisses du pouvoir romain, ou de Socrate buvant la ciguë grecque par devoir, a refusé de se soumettre à l'Empire et s'est réfugié dans une caverne (!), où il a fondé, avec son fils, la tradition spéculative juive dite cachée, soit ce que Heidegger aurait appelé les « concepts fondamentaux ». Et vous comprenez à présent que vu sous cet angle, entre la thèse de Lacoue-Labarthe et celle de Badiou, qu' a priori tout oppose, il y a, au fond, l'espace d'un cheveu, puisque l'un et l'autre opposent à Heidegger 
que «juif » est un nom de la pensée, et que tout penseur véritable est comme voué à ce nom. Citons Badiou, qui écrit dans ses Portées du mot « juif », au sujet de l'étatisation du nom « juif » : «Un "goy" le dit avec passion : sauver le nom "juif" lui est essentiel, parce que c'est de sa propre détermination conceptuelle et agissante qu'il s'agit. » La question n'est donc pas d'opposer l'antiphilosophe et le philosophe, mais de se risquer sur le chemin de la pensée, à la rencontre du mot « juif ».

On pourrait conclure que, depuis une trentaine d'années, l'intellectualité française a été la scène d'une extraordinaire intuition quant à la signifiance de l'existence juive, avec d'une part l'œuvre de Duras, la réception de l'œuvre poétique de Celan en France (remarquablement traduite par Martine Broda), la pensée de Lévinas ou la méditation de Lacoue-Labarthe, et d'autre part ce que j'appellerai, non sans provocation, le «platonisme juif » de Badiou, au sens où chez lui la littéralité mathématique instruit la rupture avec le spiritualisme en philosophie, dont tous les chemins mènent à Rome, de même que la systématicité talmudique opère une sorte de rupture épistémologique avec l'interprétation religieuse des textes et ses questionnements infinis. (C'est du reste pourquoi il est amusant de voir Badiou critiquer le littéralisme de Moïse et lui préférer l'universalisme de Paul, véritable héritier d'Abraham selon lui, alors même que, dans sa pratique de la philosophie, Badiou me paraît être un lointain disciple de Moïse plutôt que de Paul.) Et il me semble que cela correspondrait d'une part, pour ce qui est de l'espace non-philosophique ou poétique, voire antiphilosophique, à une intuition de la kedousha d'Israël, qu'on pourrait traduire par la signifiance d'Israël, au sens où Celan écrit : « Dis : Jérusalem est », mais aussi au sens où la poétesse russe Marina Tsvetaiéva, citée par Celan en exergue d'un poème de La rose de personne, écrit : "Tous les poètes sont des youtres ». Et cela correspondrait d'autre part, dans l'espace cette fois proprement philosophique, rationaliste, autrement dit platonicien, à une intuition de la hokhma d'Israël, qu'on pourrait donc traduire par l'Idée. Mais bien sûr, par Israël, je n'entends nullement ici l'État d'Israël, qui n'est autre, à mon sens, qu'une contingence hégélienne de l'Histoire, laquelle n'a donc, en tant que telle, que fort peu à voir avec l'essentiel. À l'inverse, si l'on tient que le sionisme étatique est la seule réponse qui soit à la question de l'existence signifiante, et en Idée, des Juifs, on en vient à délégitimer toute autre pensée qui ne serait pas étatique, autrement dit toute pensée qui ne serait pas petitement hégélienne. Et 
dans ce type de procès en légitimité, rien de tel que d'user et d'abuser du qualificatif d' " antisémite », quitte non seulement à occulter, mais à disqualifier l'apport capital, par endroits fulgurant, de la pensée contemporaine de langue française sur cette question des Juifs. Or il me semble que l'existence signifiante par soi des Juifs a justement à voir avec une forme d'émancipation humaine essentiellement hétérogène à ce qui nous tient aujourd'hui lieu de philosophie naturelle, j'entends l'idéologie des sociétés occidentales dites « démocratiques ».

Basile Dewez : Revenons à Heidegger. Vous montrez, par votre longue analyse d'Eichmann à Jérusalem, que Hannah Arendt, d'une certaine façon, justifie le silence de Heidegger sur l'extermination des Juifs, puisqu'elle conclut que le motif antisémite du génocide est « contingent », et que l'essentiel serait bien, comme l'expose Heidegger dans sa phrase mettant en rapport l'extermination par chambres à gaz et l'industrialisation de l'agriculture, non pas la «question juive», mais la « question de la technique ». En quoi penser Auschwitz suppose-t-il de penser la question de la technique ? Et Heidegger se serait-il exclu de la philosophie en prononçant une telle phrase, comme le prétend Emmanuel Faye?

Ivan Segré : Disons que, selon Heidegger, l'oblitération de la parole inaugurale grecque et le règne planétaire de la technique sont une seule et même chose, en ce sens que le second est l'aboutissement du premier. Or, toujours selon Heidegger, il est absolument illusoire de croire que les exterminations de masse sont le produit d'une volonté humaine immorale que l'on pourrait éduquer, corriger ou circonvenir par l'enseignement de quelque loi morale, ou l'établissement de quelque bonne gouvernance. En effet, selon lui, la volonté humaine, philosophique, politique, sociale, etc. se croit libre alors qu'elle est en réalité surdéterminée, pourrait-on dire, par le règne planétaire de la technique. Auschwitz, selon Heidegger, loin d'être un événement exceptionnel, singulier, est une réalité quotidienne, celle de l'assujettissement de l'homme au règne de la technique. Dans "Le mot de Nietzsche, "Dieu est mort" ", Heidegger écrit : "Car ce que celui-ci donne à penser à la pensée, n'est pas quelque sens ultime et très caché, mais quelque chose de proche : à savoir le plus proche, que nous outrepassons constamment parce qu'il n'est précisément que le plus proche. Par un tel passer-outre, nous accomplissons constamment, sans 
y prêter attention, le meurtre de l'être de l'étant. » Penser Auschwitz de manière essentielle, autrement dit en allant au-delà des considérations factuelles, empiriques, qui elles ne relèvent pas d'une médiation sur l'essentiel, c'est donc, selon Heidegger, penser le meurtre de l'étant, que nous accomplissons constamment. Et Heidegger ne cesse d'enseigner après Auschwitz qu'il n'est d'autre issue au destin de l'Occident, d'autre avenir à la pensée occidentale que de ressaisir le sens de la parole inaugurale grecque.

J'ai montré dans mon ouvrage de quelle manière les comptes-rendus du procès Eichmann par Arendt, parus aux États-Unis en 1963, sont marqués par l'influence de Heidegger, influence dont on retrouve la trace jusque dans les formulations d'Arendt sur Auschwitz. Ainsi Heidegger, dans sa fameuse phrase, parle d'une «fabrication de cadavres dans les chambres à gaz ». Or Arendt reprend cette même expression dans un entretien diffusée par la télévision allemande en 1964, donc immédiatement après l'écriture de son Eichmann à Jérusalem, et en allemand : « la fabrication de cadavres dans les chambres à gaz ». Cette reprise à la lettre de la formulation de Heidegger est évidemment significative, fûtelle inconsciente, ou précisément parce qu'elle l'est. Et elle est d'autant plus significative qu'Emmanuel Faye a au moins le grand mérite d'avoir attiré notre attention sur ce point, lui qui conclut, en découvrant une telle expression dans la bouche de Heidegger, prononcée lors d'une conférence datant de 1949, qu'il s'est par là même « exclu de la philosophie ». Emmanuel Faye fait de la philosophie comme Monsieur Jourdain fait de la prose, sans le savoir. En effet, « la fabrication de cadavres dans les chambres à gaz » est une formulation inattendue, singulière, et qui en appelle donc à une certaine pensée de l'événement, d'où ceci qu'en s'exprimant de la sorte, Arendt fait signe vers Heidegger, vers sa pensée de l'événement Auschwitz, outre qu'elle s'exclut donc de la philosophie en contribuant par là même à « la déshumanisation par le nazisme des victimes des camps d'extermination ». Mais laissons Monsieur Jourdain à sa prose, et revenons à la formulation de Heidegger. S'il s'est agi, à Auschwitz, d'une «fabrication de cadavres », c'est parce que le processus de mise à mort était un processus industriel, ou du moins calqué, instruit par le processus industriel de production de masse. Or, selon Heidegger, c'est ce fait qui est décisif pour la pensée, puisque se révèlerait par là même que le nihilisme nazi a sa racine, sa radicalité pourrait-on dire, dans le règne planétaire de la technique, et non dans une idéologie raciale 
qu'une bonne vieille morale humaniste pourrait circonvenir. Voilà ce que nous enseignerait Heidegger par sa fameuse phrase. Qui n'en reste pas moins scandaleuse, objecte pourtant Lacoue-Labarthe. Pourquoi ? Non pas parce que Heidegger se serait ainsi exclu de la philosophie conçue dans les limites de la simple raison d'Emmanuel Faye, mais pour une toute autre raison, à savoir que Heidegger aura omis de s'interroger sur le fait qu' on s'en est pris aux Juifs. Or, soutient Lacoue-Labarthe dans sa Fiction du politique, « ce n'est par aucune sorte de hasard que ceux que l'on voulait anéantir étaient les témoins, dans cet Occident-là, d'une autre origine du Dieu qui y avait été vénéré et pensé ». Quant à Faye, tout cela lui passe largement au-dessus de la tête, puisqu'il ne relève absolument pas le silence de Heidegger sur ce point. En effet, selon Emmanuel Faye, le scandale de la phrase de Heidegger ne réside pas dans l'omission du mot « juif », comme le pense Lacoue-Labarthe, mais dans « le fait qu'il assimile le meurtre programmé de millions d'êtres humains à une industrie destinée à fabriquer des cadavres, comme si les SS avaient eu l'intention de produire mécaniquement des cadavres comme on fabrique du sucre, en anéantissant des millions d'hommes, de femmes et d'enfants complètement retranchés par eux de l'espèce humaine ». D'où la question que, pour ma part, je vous pose : qui, de Heidegger ou d'Emmanuel Faye, s'est exclu de la philosophie en prononçant une telle phrase ?

Basile Dewez : Votre second ouvrage, La réaction philosémite, dans lequel vous critiquez un certain nombre d'intellectuels français engagés d'après eux dans la « défense du sionisme » et la « lutte contre l'antisémitisme », a pour sous-titre La trahison des clercs. Pourquoi cette référence au livre de Benda ?

Ivan Segré : Je me suis proposé de retourner la formule de Julien Benda, qu'il faut lire, sous ma plume : la trahison du judaïsme par des Juifs convertis au cléricalisme intellectuel français. De la même manière, « philosémite » est à entendre sur le mode de l'antiphrase, de même que « réaction philosémite » opère sur le modèle poétique de l'oxymore, la réaction idéologique et le philosémitisme étant selon moi contradictoires. Mes analyses de textes n'ont d'autre enjeu que d'éclairer le lecteur sur le sens véritable du titre ( Réaction philosémite ») et du sous-titre ( $\mathrm{La}$ trahison des clercs »), en montrant, en démontrant que des intellectuels comme Shmuel Trigano, Alexandre Adler, Alain Finkielkraut, Emmanuel 
Brenner (alias Georges Bensoussan) ou encore Pierre-André Taguieff, bien loin de s'inscrire dans une pensée sioniste ou de se soucier de la «lutte contre l'antisémitisme », renouent en fait avec le mot d'ordre réactionnaire et xénophobe de la « défense de l'Occident ». Car, comme je l'indiquais plus haut, la défense de l'État d'Israël ne comptera bientôt pour rien dans cette affaire, l'essentiel étant de produire une argumentation qui désigne le « musulman » et le « gauchiste» comme des ennemis de la démocratie occidentale. Pour exemple, je cite dans mon livre un texte d'Alexandre Adler qui, dans l'Odyssée américaine (Grasset, 2004), nous fait part de ses visions prophétiques sur l'avenir de l'État d'Israël, et conclut : «L'une des frontières de l'Amérique se situera bientôt sur le Jourdain, mais ce sera une frontière électronique, cybernétique, balistique, laissant aux gardes-frontières juifs et druzes le soin de faire la police au sol, face à un État palestinien qui, étroitement relié aux forces nationalistes et sunnites les plus résolues, fera toujours partie du problème et non de la solution, au Moyen-Orient. » Il me semble que les visions prophétiques d'Alexandre Adler font clairement apparaître qu'en guise de défense d'Israël, ces intellectuels défendent une conception du judaïsme qui n'a d'autre consistance que celle-ci : des « gardes frontières juifs et druzes » chargés de faire « la police au sol » sur la frontière américaine du Jourdain. Vous voyez que, pour réduire à néant l'argumentaire prétendument juif, sioniste ou philosémite de ces intellectuels, il n'y a finalement pas grandchose à faire : il suffit de les citer. 